\title{
Center or side: biases in selecting grasp points on small bars
}

\author{
Vivian C. Paulun · Urs Kleinholdermann • \\ Karl R. Gegenfurtner · Jeroen B. J. Smeets • \\ Eli Brenner
}

Received: 17 July 2013 / Accepted: 25 February 2014 / Published online: 18 March 2014

(C) Springer-Verlag Berlin Heidelberg 2014

\begin{abstract}
Choosing appropriate grasp points is necessary for successfully interacting with objects in our environment. We brought two possible determinants of grasp point selection into conflict: the attempt to grasp an object near its center of mass to minimize torque and ensure stability and the attempt to minimize movement distance. We let our participants grasp two elongated objects of different mass and surface friction that were approached from different distances to both sides of the object. Maximizing stability predicts grasp points close to the object's center, while minimizing movement costs predicts a bias of the grasp axis toward the side at which the movement started. We found smaller deviations from the center of mass for the smooth and heavy object, presumably because the larger torques and more slippery surface for the heavy object increase the chance of unwanted object rotation. However, our righthanded participants tended to grasp the objects to the right of the center of mass, irrespective of where the movement started. The rightward bias persisted when vision was removed once the hand was half way to the object. It was reduced when the required precision was increased. Starting the movement above the object eliminated the bias. Grasping with the left hand, participants tended to grasp the object to the left of its center. Thus, the selected grasp points seem to reflect a compromise between maximizing stability by grasping near the center of mass and grasping
\end{abstract}

V. C. Paulun $(\bowtie) \cdot$ U. Kleinholdermann $\cdot$ K. R. Gegenfurtner Department of Experimental Psychology, University of Gießen, Otto-Behaghel-Str. 10F, 35394 Gießen, Germany e-mail: Vivian.C.Paulun@psychol.uni-giessen.de

V. C. Paulun · J. B. J. Smeets · E. Brenner

Faculty of Human Movement Sciences, MOVE Research Institute, VU University, Van der Boechorststraat 9,

1081 BT Amsterdam, The Netherlands on the side of the acting hand, perhaps to increase visibility of the object.

Keywords Grasp point selection - Movement distance · Prehension · Weight · Vision · Minimum energy · Minimum torque

\section{Introduction}

To manipulate or interact with objects in the environment, humans often use a precision grip of index finger and thumb. An elementary aspect of the way we grasp an object is the selection of grasp points; i.e., the locations at which the digits touch the object in order to grasp it. For most objects, there are a huge number of possible grasp points. Depending on the object's properties, many of them will not lead to a successful (i.e., stable) grip and many other will be inconvenient.

Several basic constraints have been argued to play a role in grasp point selection. For a stable grip, it is necessary that the two digits touch the object at positions that lie within the friction cones, a constraint that is referred to as force closure (Blake 1995). Furthermore, the object's center of mass is important for the selection of grasp points. If one holds an object at its center of mass, it will be balanced and no further torque will act on it. If the axis between the two digits (the grasp axis) does not pass through the object's center of mass, more force may be needed to hold the object in equilibrium, as the additional torque could otherwise lead to a rotation of the object. Previously, it has been shown that humans can accurately judge the location of the center of mass in various flat objects and use object symmetry as a reliable indicator for this (Bingham and Muchisky 1993). Lederman and Wing (2003) extended these results 
by showing that individuals choose grasp points that let the grasp axis pass near or through the center of mass. For asymmetrical objects, the position of the center of mass is presumably more difficult to determine and such objects were grasped further off-center.

Besides force closure and the perceived position of the center of mass, several other factors have been reported to determine the selection of grasp points; these include properties of the object that is to be grasped as well as the general settings in which the movement takes place. Choice of grasp points is influenced by the size of the object (Paulignan et al. 1997), its shape (Cuijpers et al. 2004; Kleinholdermann et al. 2007), its mass, with increased mass leading to grasp axes that pass more closely through the center of mass (Eastough and Edwards 2007), as well as the object's material, which determines the friction coefficient. Object parts with higher friction are preferred for grasping (Wing and Lederman 2009), while lower friction requires more precision and allows less tolerance of grasp errors, resulting in less deviations of grasp points from the center of mass (Fikes et al. 1994).

Furthermore, the selection of grasp points changes with the object's orientation (Cuijpers et al. 2004), its location in space (Paulignan et al. 1997), the direction of the movement (Roby-Brami et al. 2000) and also through prior perceptual experience (Hesse et al. 2008). On the other hand, grasp points do not change with a change in the hand's path, as imposed by the existence of an obstacle, which might indicate that grasp points are selected prior to planning the movement toward these selected locations on the object (Voudouris et al. 2010). As evident from the determining factors mentioned above, grasp point selection depends strongly, but not exclusively on information acquired through vision.

Endo et al. (2011) showed that grasp point selection is influenced by a multimodal representation of the object and its center of mass. In their study, a shift of the object's center of mass caused a shift of the grasp axis toward the center of mass if there was a reliable visual cue for center of mass position or if haptic feedback was given in the absence of a visual cue. Grasp point selection might also be influenced by factors of convenience, like for instance approaches of end-state comfort (Rosenbaum et al. 1990) or other subjectively preferred postures of the hand, as indicated by the observation of a natural grasp axis (Lederman and Wing 2003). Kleinholdermann et al. (2013) successfully modeled human grasp point selection by combining this subjectively preferred grasp axis with attempts to fulfill force closure, minimize torque and minimize movement distance.

In addition to the above-mentioned determinants, other factors may influence grasp point selection. It is for instance reasonable to hypothesize that humans aim to perform grasping movements economically, i.e., to minimize the amount of work and thus the expenditure of energy. This has already been shown for different kinds of human movements such as walking and running (Alexander 1991) or jumping (Vanrenterghem et al. 2008). In addition to locomotion, the role of energetic costs has also been investigated in various arm movements such as pointing and reaching. Energy minimizing models are consistent with the choice of final postures in a simple pointing task (Soechting et al. 1995) and with arm trajectories in such tasks (Alexander 1997). Taniai and Nishii (2008) showed that trajectories of reaching movements of various durations can be modeled well by trajectories that aim to minimize total expenditure of energy under the effect of signal-dependent noise, as proposed by Harris and Wolpert (1998). Some authors, on the other hand, argue (based on a combination of experiment and model calculation) that minimizing energy is not very relevant for goal-directed arm movements (Kistemaker et al. 2010). Direct measurement of energy consumption during motor learning seems to suggest that reducing energy consumption is important for arm movements (Huang et al. 2012), but energy minimization alone is unlikely to account for the chosen arm movements. Many authors therefore propose a mixed or hybrid explanation, in which energy minimization plays a role, but is combined with other factors such as smoothness at the level of joints and avoiding pain (Berret et al. 2011; Todorov 2004; Zelik and Kuo 2012). Elliott et al. (2004) showed that energy expenditure can be optimized together with speed and accuracy through motor learning and strategic behavior across subsequent aiming movements. Taken together, results reported in the literature indicate that energy saving principles play an important role in human motor control, although the extent to which this factor influences motor control is controversial.

The aim of the present study was to investigate factors influencing grasp point selection by bringing them into conflict. For this purpose, we asked participants to grasp bar-like objects with a precision grip of index finger and thumb, starting from several different positions at various distances to the target object. On the one hand, it can be expected that individuals seek to grasp the object at its center of mass to reduce torque and thus the amount of force needed to lift the object; on the other hand, from the literature mentioned above, it seems likely that humans also take other energetic costs into account when choosing appropriate grasp points. Thus, individuals might prefer to cover a shorter distance and thus show a bias in grasp point selection away from the center of mass and toward the start point of the movement. Such an effect should be more pronounced when grasping an object of lower mass, for which deviations from the center of mass will lead to a smaller increase in torque than for a heavier object. Similarly, 
objects with higher surface friction are less prone to rotate or slip and should thus therefore permit a more off-center grip. For this purpose, two different objects were used in the experiment: a light one with a rather rough surface and a heavier one that was fairly smooth. Both were easy to distinguish visually as well as haptically. The majority of studies on grasp point selection used only spherical or cylindrical objects as stimuli. Such objects are in some sense special as grasp points that are located on opposing sides of the object will always lead to a grasp axis through the center of mass. Rectangular, bar-like objects were used in the present study because the grasp axis can be shifted in one direction without changing force closure.

\section{Main experiment}

Methods

\section{Participants}

Twenty-one right-handed participants took part in our experiment, 14 females and seven males. All of them were researchers or graduate students of the VU University in Amsterdam. The participants' ages ranged between 23 and 41 years $(M=28$ years $)$. All participants were naïve to the aim of the study and gave their informed consent prior to participation. The study is part of a research program that has been approved by the ethics committee of the Faculty of Human Movement Sciences.

\section{Stimuli}

Two bar-like objects served as stimuli, see Fig. 1. Both had the same size of $10 \times 3 \times 1 \mathrm{~cm}$. One object was made of polished polyoxymethylene (POM), a black plastic material, with a rather smooth surface, and had a mass of $42.3 \mathrm{~g}$ and a density of $1.41 \mathrm{~g} / \mathrm{cm}^{3}$. The second, lighter $(0.8 \mathrm{~g})$ object was made of light green polystyrene with a density of $0.0267 \mathrm{~g} / \mathrm{cm}^{3}$ and had a rough surface.

\section{Setup}

Participants were seated at a table. Target objects were placed on the table with the wide elongated side on the table, oriented fronto-parallel (see Fig. 2). The object's center was at a distance of $25 \mathrm{~cm}$ from the near edge of the table (from the participant's viewpoint). Five different start positions were marked with little plastic knobs, each $11 \mathrm{~mm}$ in diameter and $5 \mathrm{~mm}$ high. Three start positions were located to the right side of the object, 10, 20 and $30 \mathrm{~cm}$ from the target's center. Two start positions were placed at its left side at distances of 20 and $30 \mathrm{~cm}$ from its

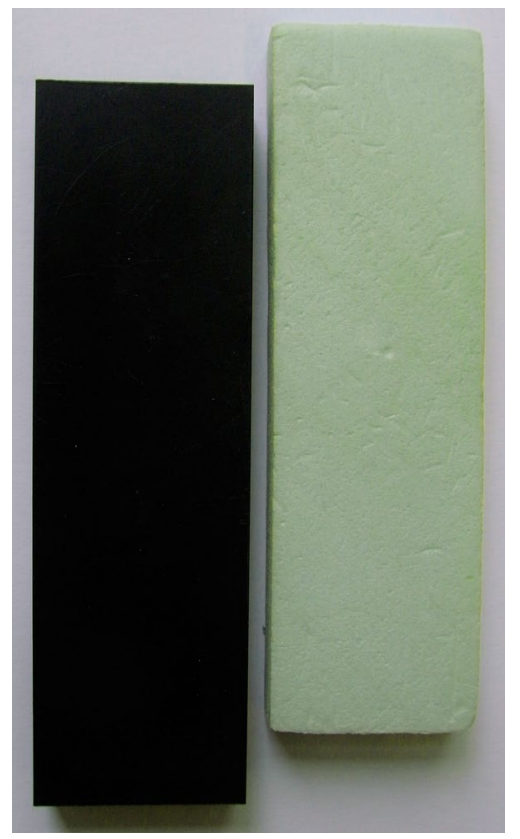

Fig. 1 The two objects used in this study. One object was made of black POM (left). The other one was made of light green polystyrene (right) (color figure online)

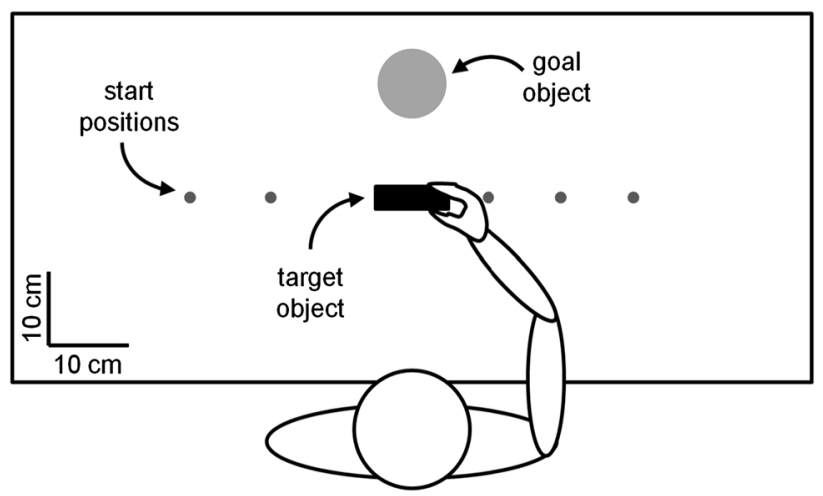

Fig. 2 Top view of the experimental setup. Participants sat in front of the table and were asked to start their movement at one of the indicated start positions, grasp the object by its elongated sides with a precision grip (between index finger and thumb) of their right hand and carry it to the goal object to place it onto it. The Optotrak camera was located to the left of the table

center. A start position $10 \mathrm{~cm}$ to the left of the target center was not used, as the object would have been under the right hand when grasping. Participants were asked to grasp the object with their right hand and lay it down on a metal cylinder with a diameter of $9 \mathrm{~cm}$ and a height of $3 \mathrm{~cm}$, which was placed behind the target. The distance between the cylinder's center and the center of the target object was $18 \mathrm{~cm}$.

Grasping movements were recorded with an Optotrak 3020 infrared tracking system with a sampling frequency of 
$200 \mathrm{~Hz}$. The Optotrak camera was located to the left of the table (from the participants' viewpoint). Sets of three infrared markers forming a rigid body were attached to the nails of index finger and thumb to record movements of these two digits (as depicted in Fig. 1 of Voudouris et al. 2010). The finger tips were calibrated in relation to the three markers. For this purpose, participants were asked to grasp a wooden bar $(5 \times 2.5 \times 1 \mathrm{~cm})$ with a precision grip at two points of which the exact positions in space had been measured prior to calibration. Participants wore PLATO liquid crystal glasses (Milgram 1987), which provide full vision in their clear state and become milky white and translucent in their closed state.

\section{Procedure}

Participants were instructed to grasp the object by its elongated sides with a precision grip, using the index finger and thumb of their right hand, carry it to the metal cylinder and place it on top of it. No further instruction was given on how or where to grasp the target objects. Prior to each trial, the experimenter indicated the start position by mentioning a number between 1 and 5. Each number was assigned to a start position (increasing from left to right). As soon as the participant's index finger and thumb reached the indicated start position, the glasses turned translucent. The experimenter then placed one of the two objects at the target position. Small stripes of tape were used to indicate the exact position; the edges of the tape physically guided the object to this desired position. To verify the precision of this method, we calculated the deviation of the grasp center in depth (i.e., in the dimension orthogonal to our dependent measure, see below) which should be constant. The standard deviation was less than $1 \mathrm{~mm}$, so the precision with which the objects were positioned in our study was more than sufficient. A beep signaled the start of a trial, with the glasses turning transparent at the same moment. The glasses remained in this state for $2 \mathrm{~s}$ during which participants completed the task. Five to ten practice trials were done prior to the experiment, so that each participant had completed five trials correctly in the sense that the object was grasped by the elongated sides and the whole movement was completed within the $2 \mathrm{~s}$. Data of these practice trials were not used for data analysis. One hundred trials were then completed with each participant. Ten trials for every combination of the two objects and five start positions were conducted in random order.

\section{Data analysis}

The parameters we were most interested in were the initial contact positions of index finger and thumb with the object. The moment of initial contact with the object was defined as the data frame in which the vertical position of either index finger or thumb was lowest (i.e., closest to the table), while at least one of the digits was not further away from the object's center than $4 \mathrm{~cm}$. The midpoint of the grasp axis (the line connecting index finger and thumb) was defined as the grasp center. As we were mainly interested in its deviations from the object's center of mass, we subtracted the horizontal coordinates of the grasp center from the horizontal coordinates of the object's center. Thus, a value of zero would indicate that grasp and object center were equal; negative values indicate a deviation of the grasp center to the left; positive values indicate a deviation to the right. We calculated the mean grasp center and its standard deviation for each participant, object and start position. A one-sample $t$ test was used to test whether the grasp center differed significantly from zero, i.e., from the object's center. To evaluate the influence of the different objects and start positions on grasp point selection, we used 2 (objects) $\times 5$ (start positions) repeated measures ANOVAs for the mean grasp center and its variability.

Furthermore, a 2 (objects) $\times 5$ (start positions) repeated measures ANOVA was used to compare movement durations and maximum grip apertures (MGA). Movement duration was defined as the time between the start of the movement, for which a velocity criterion of $0.025 \mathrm{~m} / \mathrm{s}$ had to be exceeded, and the moment at which the digits initially touched the object as defined above. MGA was defined as the maximum distance between the two digits during the movement. Both measures were calculated on a trial by trial basis.

If there was a violation of sphericity in any of the ANOVAs, we used a Greenhouse-Geisser correction. Welch's $t$ tests with a Bonferroni correction were used in all cases of multiple comparisons. Statistical analysis was performed using the statistical package R (R Development Core Team 2011).

Results

\section{Grasp point selection}

The mean grasp center was not aligned with the object's center of mass: It was shifted $5.5 \pm 6.2 \mathrm{~mm}$ (all values given as mean \pm between subject $\mathrm{SD}$ ) toward the right side of the object $(t(20)=4.00, p<.001)$. The 2 (objects) $\times 5$ (start positions) repeated measures ANOVA revealed that this effect was significantly stronger for the light $(6.5 \pm 7.5 \mathrm{~mm})$ than for the heavy object $(4.5 \pm 6.1 \mathrm{~mm})$ as indicated by a main effect of the factor object, see Fig. 3a. There was neither a significant main effect of the start position, nor an interaction between the factors. Results of all ANOVAs calculated for the main experiment are summarized in Table 1. 

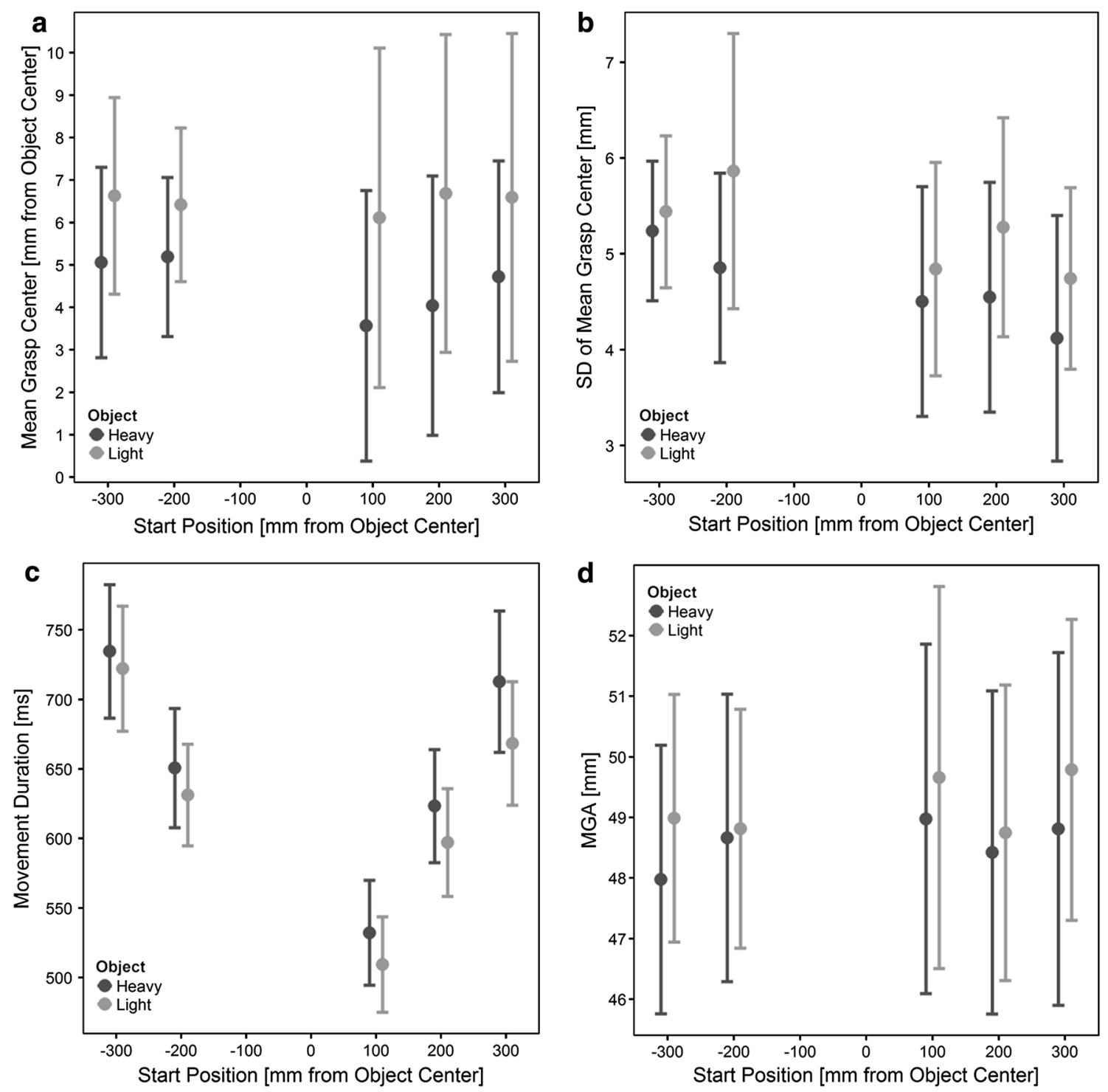

Fig. 3 Results of the main experiment. Data are averaged across participants. Error bars show $95 \%$ confidence intervals (calculated between participants). Mean grasp center (a), (within participant)

standard deviation of the grasp center (b), movement duration (c) and MGA (d) for different objects and start positions
Table 1 Results of repeated measures ANOVAs of the main experiment

\begin{tabular}{llllllll}
\hline Measure & Mean & SD & Factor & $d f_{1}^{\mathrm{a}}$ & $d f_{2}^{\mathrm{a}}$ & $F$ & $p$ \\
\hline Mean grasp & \multirow{2}{*}{$5.5 \mathrm{~mm}$} & \multirow{2}{*}{$6.9 \mathrm{~mm}$} & Object & 1 & 20 & 23.10 & $<.001$ \\
Center & & & Start position & 1.42 & 28.43 & 0.47 & .57 \\
& & & Interaction & 2.79 & 55.78 & 0.89 & .45 \\
SD of grasp & \multirow{2}{*}{$4.9 \mathrm{~mm}$} & \multirow{2}{*}{$2.6 \mathrm{~mm}$} & Object & 1 & 20 & 6.18 & .02 \\
Center & & & Start position & 2.69 & 53.85 & 2.61 & .07 \\
& & & Interaction & 2.60 & 52.05 & 0.45 & .69 \\
Movement duration & \multirow{2}{*}{$638 \mathrm{~ms}$} & \multirow{2}{*}{$121 \mathrm{~ms}$} & Object & 1 & 20 & 38.68 & $<.001$ \\
& & & Start position & 4 & 80 & 143.92 & $<.001$ \\
& & & Interaction & 4 & 80 & 2.19 & .08 \\
MGA & \multirow{3}{*}{$5.8 \mathrm{~mm}$} & Object & 1 & 20 & 4.90 & .04 \\
& & & Start position & 2.42 & 48.30 & 0.861 & .45 \\
& & & Interaction & 4 & 80 & 0.91 & .46 \\
\hline
\end{tabular}

${ }^{a}$ Greenhouse-Geiser corrected $d f \mathrm{~s}$ if sphericity violated 
Besides a larger right shift of the grasp center for the lighter object, the variability of the grasp center was also larger for this object. The 2 (objects) $\times 5$ (start positions) repeated measures ANOVA showed a significant main effect of object type on the (within participant) standard deviation of the grasp center. The standard deviation of the grasp center was higher for the light $(5.2 \pm 2.57 \mathrm{~mm})$ than for the heavy object $(4.65 \pm 2.55 \mathrm{~mm}$; see Fig. 3b). Again, there was no significant main effect of the start position, but we do see a trend $(p=.07)$ for the standard deviation to be larger when the hand starts on the left. This could be because the hand occludes the object for some time when it approaches from the left. There were no significant interactions between the factors.

Thus, analysis of the grasp center showed that participants did not grasp the objects at their center of mass, as would be expected if only taking stability constraints into account. Interestingly, the grasp center was on average shifted to the right of the object's center irrespective of where the movement started. This bias was stronger, and grasp points were more variable for the light object.

\section{Movement duration}

The 2 (objects) $\times 5$ (start positions) repeated measures ANOVA revealed a significant main effect of start position on movement duration. Expectably, the larger the distance between object and start position of the movement, the longer the movement duration, whereby the movement duration was significantly longer for equidistant start positions when starting on the left (see Fig. 3c). Subsequent paired-samples $t$ tests showed that differences between all levels of this factor were significant (all $p<.001$ ). Interestingly, there was also a significant main effect of object on movement duration. Movement duration was longer when approaching the heavy $(650 \pm 124 \mathrm{~ms})$ compared with the light object $(625 \pm 116 \mathrm{~ms})$. There was a tendency for this difference to be larger when the hand started further to the right ( $p=.08$ for the interaction between the two factors).

\section{$M G A$}

The MGA was larger for the light $(49.2 \pm 5.7 \mathrm{~mm})$ compared with the heavy object $(48.6 \pm 6.0 \mathrm{~mm})$, see Fig. $3 \mathrm{~d}$. This resulted in a significant main effect of object in the 2 (objects) $\times 5$ (start positions) repeated measures ANOVA $(p<.05)$. This ANOVA showed neither a significant main effect of start position nor an interaction between the two factors.

\section{Discussion}

The goal of our main experiment was to investigate the relative contributions of grip stability and movement energy saving constraints in grasp point selection by manipulating the mass and surface as well as the distance of target objects. Under these circumstances, we see no evidence that individuals minimize energetic costs when reaching to grasp. Starting the movement on the left or right side of the target object did not result in a shift of the grasp axis toward that side.

We did observe an effect of the factor object on grasp point selection. The grasp center was further from the object's center and more variable across trials when grasping the light object. Grasping off-center is less costly for this object because the increase in torque with distance from the center is smaller for the lighter object. The reach to grasp movements can probably be less precise for the light object because the consequences of inaccuracy are less severe. The higher the torque acting on the object and the more slippery its surface, the more grip force is needed to prevent the object from rotating when it is lifted. Thus, grasping the smooth and heavy object closer to its center and more precisely could be considered to reflect a strategy of minimizing energetic costs, the costs of larger grip forces. However, we see no evidence of minimizing energetic costs by reducing the distance travelled by the hand. At this point, it is not possible to evaluate whether minimizing movement distance is less beneficial in terms of energy expenditure than minimizing grip forces. For that, we would have to directly measure metabolic costs.

The role of energy minimization in the control of arm movements is considered controversial in the literature (see introduction). None of those studies investigated grasp point selection. Our study shows that travelled distance is not a major issue, but whether the influence of object mass and friction should be considered in terms of the risk of dropping the object or in terms of the energy required to prevent the object from rotating is not clear, so we cannot draw strong conclusions about the role of attempting to reduce energetic costs.

The fact that grasp points were more variable for the rough and light object is in line with the observation that participants approached this object significantly faster than they did the heavy one. Type of object also had an effect on the MGA: It was larger for the rough and light object. This could be interpreted as a way to partially compensate for the higher approach speed when grasping this object (Smeets and Brenner 1999). The mass and surface properties covaried in our objects: Our heavy object had a rather smooth surface and our light object had quite a rough surface. At this point, we cannot disentangle the relative contribution of friction and mass to the observed effect of the factor object.

Overall, our data clearly show our participants' tendency to grasp the objects near the center. This confirms that grasp points are selected to improve stability (e.g., Goodale et al. 
1994; Lederman and Wing 2003). Interestingly, our data suggest another important determinant of grasp point selection. We observed a rightward bias, i.e., the grasp axis was significantly shifted to the right of the object's center. This bias was present irrespective of start position or object type. To our knowledge, such a bias has not been reported before. Lederman and Wing (2003) investigated grasp point selection for similar rectangular objects. Although not explicitly mentioned in their article, inspection of their Fig. 4 (p. 162) reveals that for such objects the grasp axis also seems to be slightly shifted to the right of the center. Kleinholdermann et al. (2007) investigated grasp point selection for trapezoidal objects. For most of their objects, they report a similar shift of the grasp axis to the right of the objects' centers. As in our experiment, their right-handed participants grasped their lighter object further to the right than they did their heavier object when picking it up. The generality of these findings suggests that selected grasp points reflect a compromise between grasping at the center to ensure stability and grasping to the right. As our main experiment did not provide information about the functional role of such a rightward bias, we conducted a control experiment in order to gain further insight into its nature. For this purpose, we manipulated our setup to create specific control conditions: start above, limited vision, small goal and left hand.

\section{Control experiment}

For a better understanding of the mechanisms and possible functions of the rightward bias, we conducted a control experiment consisting of four conditions. In our first control condition, we let our subjects start the movement above the object as opposed to from the side to test whether the bias depends on movement direction. If the bias remains in this condition, this would show that it is not related to moving laterally.

A possible function of the bias might be to increase the visibility of the target objects while they are lifted. A larger part of the target objects is visible if they are grasped further to the right with the right hand. To test this idea, we removed vision after half of the movement toward the objects so that participants could not see their hand grasping and lifting the objects. With this manipulation, grasping off-center does not increase visibility of the object while lifting it. If increased visibility is the primary function of the bias, then we should not observe such bias in this second control condition.

In a third control condition, participants were asked to gently balance the target objects onto a much smaller goal object than the one used in the main experiment. With this manipulation, we aimed to increase the required stability of the grip because the objects had to be placed more carefully. If the grip has to be more stable to place the object more precisely, grasp points should be located closer to the gravicenter of the object.

Finally, to test whether the rightward bias was related to grasping with the right hand, we let the right-handed participants grasp the objects with their left hand in a fourth control condition. If participants grasp further to the left of the target's center when using their left hand, contact points are biased to the side of the acting hand, not to the right side per se.

\section{Methods}

Nineteen right-handed participants (13 women and 6 men) with a mean age of 28 years (ranging from 23 to 41 years) took part in all four conditions of the control experiment. Ten of them had already participated in the main experiment. Task, stimuli, setup and procedure were the same as in the main experiment, except for the details mentioned below for each control condition. All variables were defined as in the main experiment. We used paired t tests to compare the influence of object type in each control condition. Likewise, individual control conditions were compared with the main experiment and, where appropriate, also with the other control conditions. For this purpose, we used Welch's t tests which correct the degrees of freedom based on the homogeneity of variance. A Bonferroni correction was used in all cases of multiple comparisons.

\section{Control condition 1: start above}

In this condition, there was a single start position above the center of the objects to test to what extent the bias is related to the lateral movement. This position was indicated by a small metal bar that was mounted $45 \mathrm{~cm}$ above the table, as in Kleinholdermann et al. (2007). After five to ten practice trials, 10 trials were completed for each object (heavy or light) in random order.

\section{Control condition 2: limited vision}

In this condition, there were only two start positions, $20 \mathrm{~cm}$ to the left and right of the center of the target objects. This distance was chosen because no effects of movement distance were observed in the main experiment and $20 \mathrm{~cm}$ was the middle distance used there. Additionally, we limited the visual information that was available during grasping. Half way through the movement to the object, when the two digits were $10 \mathrm{~cm}$ from the center of the object in the horizontal direction, the liquid crystal glasses turned translucent. As a consequence, participants were unable to see their digits reach, grasp, lift and transport the objects. This manipulation was conducted to see whether participants are biased 
to the right to increase the visibility of the object while grasping because a larger part of the object is occluded by the hand when grasping at its center. To occlude the hand at the right time, the positions of both finger tips were determined online, i.e., while the hand was moving, and the program checked whether these positions were closer to the target center than $10 \mathrm{~cm}$. The liquid crystal glasses turned translucent as soon as this critical distance was reached, and they remained in this state for the rest of the movement. After five to ten practice trials, participants completed 10 trials for every possible combination of start position (left vs. right) and object (heavy vs. light) in random order.

\section{Control condition 3: small goal}

In this condition, there were again only two start positions ( $20 \mathrm{~cm}$ to the left and right of the center of the target objects). A much smaller metal cylinder (diameter: $1.5 \mathrm{~cm}$, height: $3.5 \mathrm{~cm}$ ) was used as the goal position, onto which participants were instructed to place the target objects. Its center was at the same position as the center of the goal object in the main experiment. Participants were specifically told that they have to balance the target objects onto the small cylinder so that they do not fall. This instruction and the smaller goal were used to increase the required precision of the task because the object needs to be placed more accurately. This might make subjects grasp closer to the target's center. After five to ten practice trials, participants completed 10 trials for every possible combination of start position (left vs. right) and object (heavy vs. light) in random order.

\section{Control condition 4: left hand}

In this condition, our 19 right-handed participants were asked to grasp the objects with their left hand to see whether the bias is related to the acting hand. The participants were seated on the opposite side of the table, so that the Optotrak camera was now to their right and the setup was reversed. Again, the center of the target was $25 \mathrm{~cm}$ from the near edge of the table, and the center of the goal object was $18 \mathrm{~cm}$ behind the center of the target object. There were only two start positions located at a distance of $20 \mathrm{~cm}$ to the left and right of the target's center. As in the previous conditions, after five to ten practice trials, 10 trials were completed for every possible combination of start position (left vs. right) and object (heavy vs. light) in random order.

Results

\section{Control condition 1: start above}

Starting above the object reduced the rightward bias to an average rightward shift of $2.1 \pm 5.3 \mathrm{~mm}$, so that it no longer reached significance $(t(18)=1.73, p=.10)$, although it was not significantly different from the bias in the main experiment either $(t(73.77)=1.87, p=.07)$. Again, the grasp axis was significantly further to the right when our participants grasped the light object than when they grasped the heavy object $(t(18)=-3.82, p=.001$; see Fig. 4a). Contrary to the results of our main experiment, there were no significant differences in the variability of the grasp center for the heavy and the light object $(t(18)=0.72$; see Fig. $4 b)$, neither was there a significantly different MGA for the two objects $(t(18)=0.35$; see Fig. $4 d)$. The variability of grasp points was significantly smaller than in the main experiment $(t(31.85)=3.70, p<.001)$. When starting above the objects, our participants approached the light object significantly faster than the heavy object $(t(18)=2.93, p=.008$; see Fig. 4c). Overall, movement duration was longer than in the main experiment $(t(37.96)=-2.29, p<.05)$.

\section{Control condition 2: limited vision}

Visual information was removed on average $288 \pm 41 \mathrm{~ms}$ after movement onset, i.e., after $48 \%$ of the movement. The aperture between index finger and thumb at this point of the movement was on average $42 \pm 10 \mathrm{~mm}$, i.e., $77 \%$ of the MGA. Despite the fact that participants could not see their hand grasping, lifting and carrying the target objects, they were without exception still successful in placing the target onto the goal object. Participants showed a significant bias to grasp objects to the right of the center $(5.8 \pm 6.1 \mathrm{~mm}$, $t(18)=4.11, p<.001)$. This bias was stronger for the light compared with the heavy object $(t(18)=-4.75, p<.001$; see Fig. 4a). Overall, the bias was comparable to the bias observed in the main experiment $(t(37.80)=-0.14)$. Here, as in the start above condition, we did not observe a significant difference between the variability of grasp points $(t(18)=1.64)$ or the MGA $(t(18)=-1.54)$ for the heavy and light object (see Fig. 4b, d). The variability of grasp points was similar to the one in the main experiment $(t(32.72)=-0.67)$ and the left-hand condition $(t(18)=2.65)$ but significantly larger than in the start above and small goal condition (both $p<.001$ ). The MGA was significantly larger than in the main experiment $(t(37.37)=-3.07, p=.004)$ and in the small goal condition $(t(18)=-4.36, p<.001)$. There were no significant differences compared with the other control conditions after correcting for multiple comparisons (all $p>.04$ ). In this condition, there were no significant differences between heavy and light objects in terms of movement duration $(t(18)=2.01$; see Fig. $4 \mathrm{c})$.

Control condition 3: small goal

A significant bias was found in the small goal condition $(2.6 \pm 3.3 \mathrm{~mm}, t(18)=3.33, p=.004)$. It tended to be 

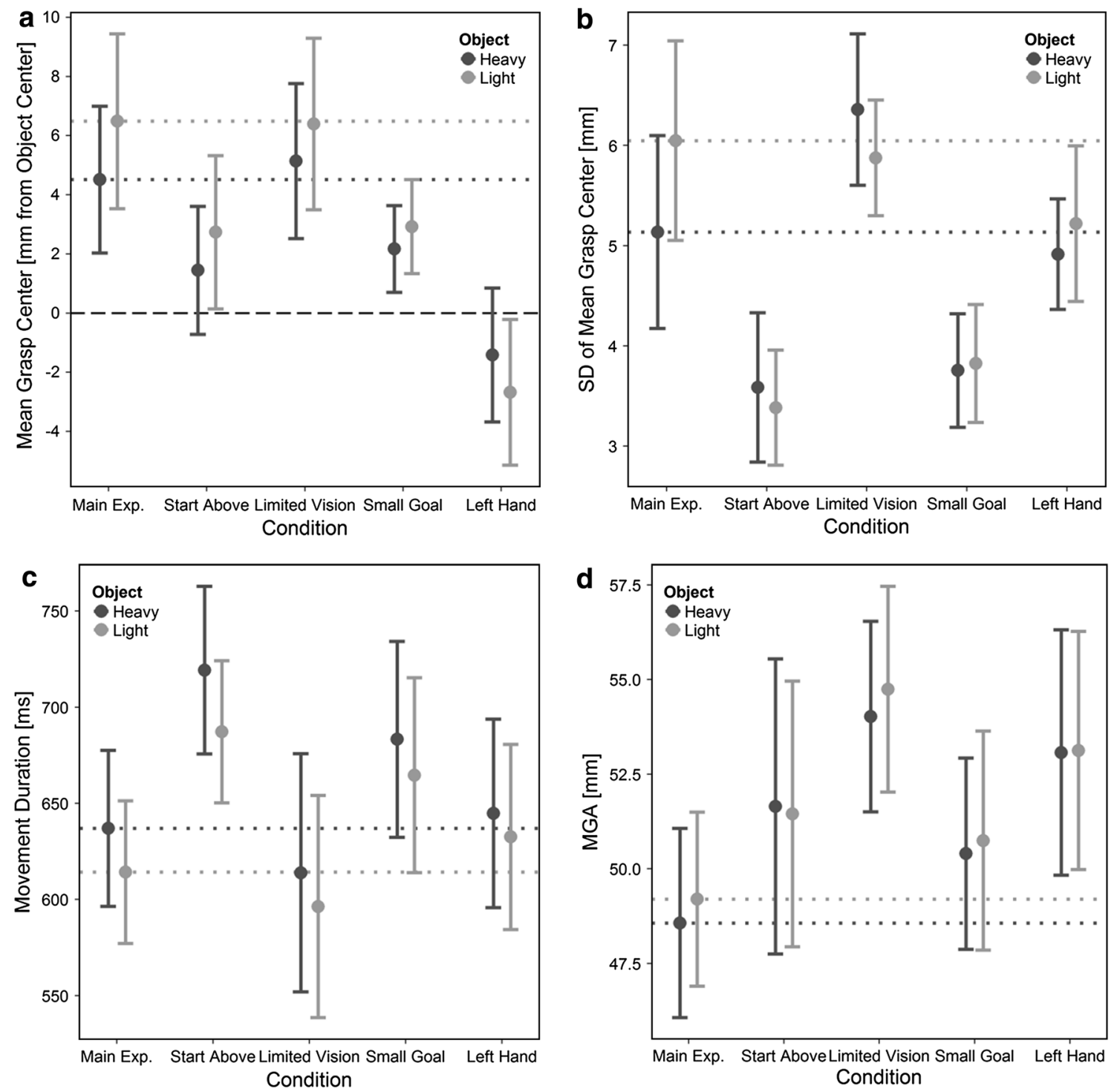

Fig. 4 Results of the control experiment. Leftmost points and dotted lines show data from the main experiment for comparison, averaged across start positions and participants. In c, only trials of the main experiment in which the start position was $20 \mathrm{~cm}$ from the object are considered. The other points show the mean values for each of the two objects for each control condition. Error bars show $95 \%$ confidence intervals (calculated between participants). a Mean grasp

smaller than the bias observed in the main experiment $(t(30.9)=1.88, p=.07)$. Participants grasped further off-center when lifting the light compared with the heavy object (see Fig. 4a); however, this comparison also failed to reach significance after Bonferroni correction which set the alpha level to $\alpha=.01(t(18)=-2.35, p=.03)$. The light object was approached faster than the heavy one $(t(18)=4.30, p<.001$; see Fig. 4c). There were no significant differences between grasping the heavy or light object in terms of grasp point variability $(t(18)=-0.35)$ or MGA $(t(18)=-0.76$; see Fig. $4 \mathrm{~b}, \mathrm{~d})$. The variability of grasp

center. The dashed line shows the object center. Positive values indicate a rightward shift of the grasp center. b Mean (within participant) standard deviation of the grasp center. c Mean movement duration. Note that the distance between start position and object center was larger in the start above condition than in all other conditions (45 rather than $20 \mathrm{~cm}$ ). d Mean MGA

points was significantly smaller in this condition than in the main experiment $(t(30.95)=3.31, p=.002)$, the limited vision condition $(t(18)=-6.82, p<.001)$ or the left-hand condition $(t(18)=-6.63, p<.001)$, but comparable to the variability when starting the movement above the object $(t(18)=0.85)$.

\section{Control condition 4: left hand}

When grasping with the left hand, our participants grasped the objects to the left of the objects' centers 
$(-2.1 \pm 5.2 \mathrm{~mm})$. However, this shift tended to be smaller than the one in the main experiment $(t(37.74)=1.89$, $p=.07)$ and thus failed to reach significance $(t(18)=1.70$, $p=.11)$. Nevertheless, the leftward shift was significantly larger when participants grasped the light compared with the heavy object $(t(18)=5.66, p<.001$; see Fig. $4 a)$. Neither the variability of grasp points $(t(18)=-1.11)$, nor the MGA $(t(18)=-0.12)$ or movement duration $(t(18)=1.63)$ differed significantly between trials with the heavy and light object (see Fig. 4b, c, d). There was no significant difference in movement duration $(t(35.32)=-0.42)$ or the variability of grasp points $(-(33.48)=1.09)$ between this condition and the main experiment. Only the MGA was significantly larger for the left hand $(t(34.31)=-2.09, p<.05)$.

\section{Hand posture}

We found a significant rightward shift of grasp points in the limited vision and small goal, but not in the start above condition. This could be due to a different posture of the hands for different movement directions, i.e., the palm of the hand might be higher in the start above condition versus more to the side in the conditions in which the objects were approached from the side. To test this, we determined the center between the three markers on a small object that was attached to the nail of each finger and projected these points orthogonally onto the (elongated) grasp axis. The angle between the line connecting the center of the marker with these projected points and the horizontal axis in the frontal plane was used as our dependent measure. This angle depends on the hand posture and is larger the higher the palm is. We calculated a mean angle for both digits and objects and each participant. As this measure depends on the position of the markers on the finger, we could only compare conditions in which the markers were attached at the same position, i.e., not taken off in between conditions as was the case for the left-hand condition. The remaining three conditions were compared with two 2 (object) $\times 3$ (condition) repeated measures ANOVAs because we could only interpret the angles meaningfully relative to one another. There were no main or interaction effects for the index finger (all $p>.05$ ). For the thumb, there was a significant difference between control conditions $(F(2,36)=5.75, p=.007)$. Angles were larger for the small goal $\left(145^{\circ} \pm 16^{\circ}\right)$ compared with the start above $\left(141^{\circ} \pm 17^{\circ}, p=.03\right)$ and limited vision condition $\left(139^{\circ} \pm 17^{\circ}, p<.001\right)$.

\section{Discussion}

With each of our four control conditions, we aimed to test a specific potential mechanistic explanations for the functional role of the effect observed in the main experiment. More specifically, the factors we explored were movement direction, visibility, stability as well as acting hand.

\section{Control condition 1: start above}

By letting our participants start the reach to grasp movement above the objects, we could test to what extent the bias is related to approaching the object from the side. In this condition, the shortest path from the start point to the object ended at its center of mass, although grasping $5 \mathrm{~mm}$ off-center only yields a 2-mm longer path. Our participants still tended to grasp the object to the right of the center, although the shift of the grasp axis was not significant. As we have shown in the main experiment that starting at the other side of the object does not affect the choice of grasping position, it is unlikely that the small differences in path length are responsible for the reduction in eccentricity in this condition. A post hoc analysis also showed that the posture of the grip was not evidently different in this condition compared with the other control conditions. There was only a difference in comparison with the small goal condition. As this effect was unique to the thumb and amounted only $4^{\circ}$, it seems as if this statistical significance does not have any practical significance in the sense of a different posture of the hand. We thus have no explanation why the effect tends to be smaller in the start above condition. When approaching the object from above, the expected horizontal variability of grasp points is smaller because the fingers approach vertically. Indeed, the intra-individual lateral variability of grasp points was significantly smaller than in the main experiment. Movement duration in the start above condition was significantly longer than in the main experiment, it actually was the longest movement duration. This is probably just because participants had to cover a longer distance (45 cm compared with $10-30 \mathrm{~cm}$ for the main experiment).

\section{Control condition 2: limited vision}

The rightward bias could reflect an attempt to increase the visibility of the object during grasping, because a larger part of the object is visible when the (right) hand is shifted further to the right. If so, we would have expected to find a reduced bias in the limited vision condition. However, we found a similar rightward shift of the grasp center as in our main experiment. Because participants did not see their hand grasping the object, it is not surprising that the variability of grasp points was highest in this condition: It was significantly higher than in the other control conditions in which participants used their right hand. However, as vision was only removed when the hand was already half way to the object, one might argue that the grasp points had 
been selected before the movement started (Voudouris et al. 2010), and limiting vision later has no effect. Movement duration was relatively short in the limited vision condition. This may be because there is no advantage in approaching the object slowly when there is no visual feedback to guide corrections. Participants might rather complete their movement quickly according to their previously selected motor plan. The relatively large MGA may reflect a strategy to compensate for some of the uncertainty that arises from making fast movements without online corrections. A similar effect on the MGA was found in another study, in which participants saw the beginning of their grasping movement but the target objects were partly hidden by an opaque occluder (Voudouris et al. 2012). In that study, the selected grasp points changed marginally in the presence of an opaque compared with a transparent or no occluder, i.e., with limited or full vision of the movement. However, with their choice of grasp points in that study, participants could determine whether they would see the selected contact locations with the object, not whether, or to what degree, they would see the object that was to be grasped itself. Even in the presence of an opaque occluder, part of the target objects was always visible to the participants in that study.

\section{Control condition 3: small goal}

The small goal condition was conducted to increase the required stability of the grasp so that the object can be placed more precisely. For a more stable grip, participants should grasp closer to the object's center. Indeed, the rightward bias that we observed in this condition, although still significant, tended to be smaller than the one seen in the main experiment. The variability of grasp points was significantly smaller than in the main experiment, it actually was the smallest of all conditions. Thus, the accuracy constraints were increased by our manipulation, confirming that the bias is due to a trade-off between stability requirements and some other factor. The movement duration was relatively long in this condition compared with the other conditions with the same distance between start point and object center. This is presumably responsible for the higher accuracy. According to Smeets and Brenner (1999), a larger MGA can also lead to higher accuracy, but MGA was relatively small in our small goal condition.

\section{Control condition 4: left hand}

We also let our right-handed participants grasp with the left hand to test whether the rightward bias that we found earlier is related to the acting right hand. If so, we should find a leftward bias when our participants grasp with their left hand. Indeed, our participants tended to grasp the object to the left of the center of mass when grasping with the left hand, although this leftward shift was not significantly different from zero. The fact that we observed a tendency to grasp to the left of the object's center when using the left hand (though it tends not to be as strong as the rightward bias when using the right hand) suggests that the bias is related to the acting hand and not to the right side per se. Otherwise, performance with the left hand was not very different from performance with the right hand in the main experiment. The main difference is that MGA tends to be relatively large. This probably accounts for the similar variability despite the unfamiliarity of using the left hand.

\section{General discussion}

The main goal of the current study was to investigate factors that may influence grasp point selection. In particular, we were interested whether participants prefer to grasp objects at their center of mass to minimize torque or to grasp off-center to save movement costs when these two strategies result in different grasp points. In the main experiment, we did not find a positional shift of the grasp axis depending on movement direction. Therefore, we have to reject the idea that cost related to movement distance was relevant in the choice of grasp position.

However, we found full support for the other part of our hypothesis: Our participants grasped further off-center when lifting the rougher light object for which the deviation from the center of mass is less costly. This effect was present in the main experiment and in three of the four control conditions, and is in line with previous research (Eastough and Edwards 2007). Besides a larger shift of the grasp center for the light object, we also consistently found a shorter movement duration for this object. Faster movements toward rougher objects have also been reported before (Fikes et al. 1994).

In all conditions we tested, participants grasped the objects near or at the center of mass. This is in line with previous research (Goodale et al. 1994; Lederman and Wing 2003) and indicates that minimizing torque is an important determinant of grasp point selection. However, there seems to be another important factor in grasp point selection that biases the grasp axis toward the side of the acting hand. A possible benefit of such placement of the fingers is that a larger part of our elongated objects was visible during lifting. This might also explain why we still found such a bias when participants were required to balance the object onto a small cylinder. On the one hand, one might argue that this should eliminate the bias, because it is particularly important to grasp the object at its center to place this part of the target object carefully onto the cylinder. We indeed found a smaller bias than in our main experiment. On the other hand, it might 
also be particularly beneficial to grasp slightly to the side to see the whole object and its center when placing it onto our target cylinder. If the attempt to increase visibility is the reason for the bias we found, why did it not disappear when we removed vision during the movement? Vision was only removed when the hand was already half way to the object, i.e., when the hand was only $10 \mathrm{~cm}$ from the object's center. Thus, even though participants did not see their hand grasp, lift and carry the objects, grasp points might have been chosen before this point in time without considering that vision would later be removed. As stated earlier, this has also been suggested in previous research (Voudouris et al. 2010). This would explain why, on average, we found a rightwards shift of the grasp axis similar to the main experiment, but with a larger within-participant variability.

Taken together, our findings suggest that grasp points are selected as a compromise between several determining factors. Our participants grasped the objects near the center of mass to maximize stability, but they additionally tended to grasp toward the side of the acting hand, possibly to increase visibility of the object while lifting it.

Acknowledgments This work was supported by a postgraduate fellowship to VC Paulun granted by the Justus-Liebig University of Gießen and by the DFG International Research Training Group IRTG 1901 "The Brain in Action-BrainAct".

Conflict of interest The authors declare that they have no conflict of interest.

\section{References}

Alexander RM (1991) Energy-saving mechanisms in walking and running. J Exp Biol 60:55-69

Alexander RM (1997) A minimum energy cost hypothesis for human arm trajectories. Biol Cybern 76:97-105

Berret B, Chiovetto E, Nori F, Pozzo T (2011) Evidence for composite cost function in arm movement planning: an inverse optimal control approach. PLoS Comput Biol 7(10):e1002183. doi:10.1371/ journal.pcbi. 1002183

Bingham GP, Muchisky MM (1993) Center of mass perception and internal frames of reference. Percept Psychophys 54(5):617-632

Blake A (1995) A symmetry theory of planar grasp. Int J Rob Res 14:425-444

Cuijpers RH, Smeets JBJ, Brenner E (2004) On the relation between object shape and grasping kinematics. J Neurophysiol 91:2598-2606

Eastough D, Edwards MG (2007) Movement kinematics in prehension are affected by grasping objects of different mass. Exp Brain Res 176:193-198

Elliott D, Hansen S, Mendoza J, Tremlay L (2004) Learning to optimize speed, accuracy, and energy expenditure: a framework for understanding speed-accuracy relations in goal-directed aiming. $\mathbf{J}$ Mot Behav 36(3):339-351

Endo S, Wing AM, Bracewell RM (2011) Haptic and visual influences on grasp point selection. J Mot Behav 43(6):427-432
Fikes TG, Klatzky RL, Lederman SJ (1994) Effects of object texture on precontact movement time in human prehension. J Mot Behav 26(4):325-332

Goodale MA, Meenan JP, Bülthoff HH, Nicolle DA, Murphy KJ, Racicotm CI (1994) Separate neural pathways for the visual analysis of object shape in perception and prehension. Curr Biol 4:604-610

Harris CM, Wolpert DM (1998) Signal-dependent noise determines motor planning. Nature 394:780-784

Hesse C, de Grave DD, Franz VH, Brenner E, Smeets JBJ (2008) Planning movements well in advance. Cogn Neuropsychol 27(7-8):985-995

Huang HJ, Kram R, Ahmed AA (2012) Reduction of metabolic cost during motor learning of arm reaching dynamics. J Neurosci 32(6):2182-2190

Kistemaker DA, Wong JD, Gribble PL (2010) The Central nervous system does not minimize energy cost in arm movements. J Neurophysiol 104:2985-2994

Kleinholdermann U, Brenner E, Franz VH, Smeets JBJ (2007) Grasping trapezoidal objects. Exp Brain Res 180:415-420

Kleinholdermann U, Franz VH, Gegenfurtner KR (2013) Human grasp point selection. J Vis 13(8):23, 1-12. doi:10.1167/13.8.23

Lederman SJ, Wing AM (2003) Perceptual judgment, grasp point selection and object symmetry. Exp Brain Res 152:156-165

Milgram P (1987) A spectacle-mounted liquid-crystal tachistoscope. Behav Res Methods Instrum Comput 19(5):449-456

Paulignan Y, Frak VG, Toni I, Jeannerod M (1997) Influence of object position and size on human prehension movements. Exp Brain Res 114:226-234

Roby-Brami A, Bennis N, Mokhtari M, Baraduc P (2000) Hand orientation for grasping depends on the direction of the reaching movement. Brain Res 869:121-129

Rosenbaum DA, Marchak F, Barnes HJ, Vaughan J, Slotta JD, Jorgensen MJ (1990) Constraints for action selection: overhand versus underhand grips. In: Jeannerod M (ed) Attention and performance XIII: motor representation and control. Erlbaum, Hillsdale, pp 321-342

Smeets JBJ, Brenner E (1999) A new view on grasping. Mot Control 3:237-271

Soechting JF, Bueno CA, Herrmann U, Flanders M (1995) Moving effortlessly in three dimensions: does Donders' law apply to arm movements? J Neurosci 15(9):6271-6280

Taniai Y, Nishii J (2008) Optimality of reaching movements based on energetic cost under the influence of signal-dependent noise. In: Ishikawa M, Doya K, Miyamoto H, Yamakawa T (eds) Neural information processing. Springer, Berlin, pp 1091-1099

Todorov E (2004) Optimality principles in sensorimotor control. Nat Neurosci 7(9):907-915

Vanrenterghem J, Bobbert MF, Casius LJ, De Clercq D (2008) Is energy expenditure taken into account in human sub-maximal jumping?-A simulation study. J Electromyogr Kinesiol 18:108-115

Voudouris D, Brenner E, Schot WD, Smeets JBJ (2010) Does planning a different trajectory influence the choice of grasp points? Exp Brain Res 206:15-24

Voudouris D, Smeets JBJ, Brenner E (2012) Do humans prefer to see their grasping points? J Mot Behav 44(4):295-304

Wing AM, Lederman SJ (2009) Points for precision grip. In: Nowak DA, Hermsdörfer J (eds) Sensorimotor control for grasping: physiology and pathophysiology. Cambridge University Press, Cambridge, pp 193-203

Zelik KE, Kuo AD (2012) Mechanical work as an indirect measure of subjective costs influencing human movement. PLoS One 7(2):331143. doi:10.1371/journal.pone.0031143 